\title{
VLHC/NLC SLOW GROUND MOTION STUDIES IN ILLINOIS
}

\author{
V. Shiltsev, J.Lach, FNAL, Batavia, IL 60510, USA; B.Baklakov, A.Chupyra, A.Erokhin, \\ M.Kondaurov, V.Parkhomchuk, E.Shubin, S.Singatulin, Budker INP, Novosibirsk, Russia 630090
}

\section{Abstract}

Since October 1999 we carry out continuous measurements of the slow ground motion on and nearby Fermilab site with a primary goal to provide experimental data for the Very Large Hadron Collider (VLHC) and Next Linear Collider (NLC) projects. Here we give a general description of the experimental set-up, present main results and discuss consequences for the colliders.

\section{INTRODUCTION}

Fig.1 shows general layout of the experimental set-up which consists of a hydrostatic level system, data acquisition stations and a PC for communication with the stations, data storage and processing. Hydrostatic level system is based on principle of equal water levels in communicating vessels. Each vessel is equipped with a capacitive sensor and a thermal sensor and linked to its neighbors by four $1 / 2$ " diameter polyethelene pipes ( 2 for water and 2 for air connection). The air circuit between the vessels has only one small opening to the atmospheric pressure. Water, being inexpensive, safe and a good electric conductor, is used as the reference fluid. However, it has a relatively large coefficient of dilatation which is temperature dependent (about $T\left[{ }^{\circ} \mathrm{C}\right] * 10^{-5} 1 /{ }^{\circ} \mathrm{C}$ for temperatures in the range $10-30{ }^{\circ} \mathrm{C}$ ). Corresponding temperature corrections are being made by computer on base of the local temperature measurements. In a $210-\mathrm{m}$ long system of water filled pipes, any perturbation results in an equilibrium state after about 2-3 minutes.

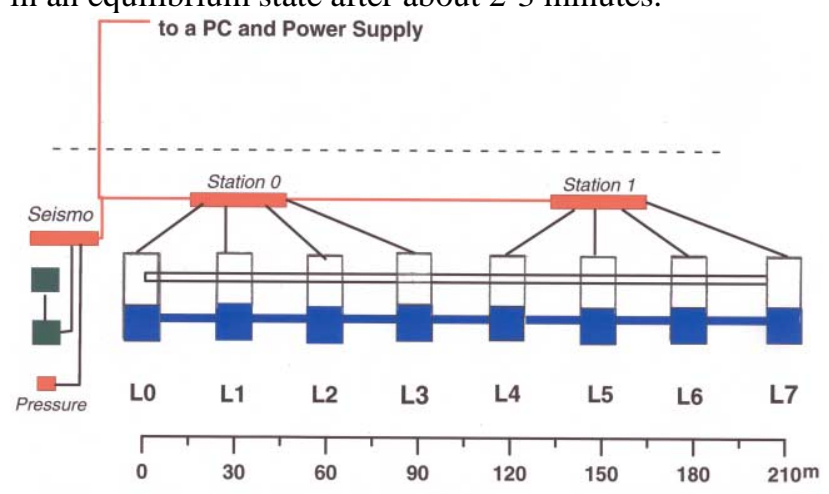

Figure 1: General layout of the slow ground motion measurement system with hydrostatic level sensors.

8 Hydrostatic Level Sensors (HLS) are developed by Fogale Nanotech (france) in cooperation with ESRF (Grenoble, France). Each consists of stainless steel vessel, capacitive sensor of displacement and platinum PT100 temperature sensor. A non contact capacitive sensor of displacement measures the vertical distance between the free water surface and a round flat electrode made of metal and ceramics. Internal electronics of HLS forms output voltage signals for the distance and temperature in range $0-10 \mathrm{~V}$. The signals are basically linear with distance and temperature with a small non-linearity which is typically a third order polynomial. Proper corrections based on the factory calibration are being taken by the computer. Each HLS is mounted on a heavy platform that is set on the ground. The altitude of each can be adjusted by using 3 screws which have a ceramic nib, for better long term stability and for electrical insulation from the ground.

Two electronic stations ( 0 and 1 in Fig.1) digitize analog signals and send data to the PC once a minute (fixed rate). Simultaneously, we measure air pressure and amplitudes of vertical and horizontal vibrations by using a system previously described in [1] ("seismo" in Fig.1). A single RG213 cable provides power to all stations (total of $40 \mathrm{~V} 2 \mathrm{~A} \mathrm{DC}$ ) and carries data flow from the stations to the $\mathrm{PC}$ and back.

To estimate the system noise, we had installed all the HLS probes side-by-side in a quiet, temperature stabilized (sealed) room in the basement of the FNAL Linac gallery for about 2 days starting 9a.m. Saturday, September 4, 1999. We found that the first difference $F D 01=(L O-L 1)$ between levels in the probe \#0 and probe \#1 varies by about $1 \mu \mathrm{m}$ with characteristic period of 1 day (1440 minutes). The same period is seen in the average temperature $T m=(\Sigma T i) / 8$ which varied by $0.08^{\circ} \mathrm{C}$ (from $26.96{ }^{\circ} \mathrm{C}$ to $\left.27.06{ }^{\circ} \mathrm{C}\right)$. The second difference $S D 0112=(L 0-L 1)-(L 1-L 2)$ also varies with 1 day period. The second difference is of a bigger interest for accelerators as it does better reflect dangerous random magnet-to-magnet movements. Another important value is the power of relative motion which occur over given time interval. For that purpose, for any function $Y(t)$ we calculate the value of dispersion $D_{-} Y(T)=\langle(Y(t+T)$ $\left.Y(t))^{2}\right\rangle$, where brackets $\langle\ldots\rangle$ stay for average over all possible pairs of data separated by time interval $T$. For the noise measurements, we found that $D \_S D 0112(T)$ is less than $1 \mu \mathrm{m}^{2}$ for time intervals up to 2 days, and is about $0.03 \mu \mathrm{m}^{2}$ for $T=1$ hour. These numbers set the system noise.

Future colliders, like VLHC[2] and NLC[3] will be very sensitive to random motion of quadrupole magnets. The characteristic time and space intervals of interest are $T=$ =weeks-years and $L=135 \mathrm{~m}$ (quad-to-quad distance) for the VLHC, and up to $1 / 2$ hour (time though to be needed for beam-based alignment system to steer the elements) and $L=10 \mathrm{~m}$ for the NLC. Our measurements cover the range of $T=3$ minutes $-1 / 2$ year and $L$ from $30 \mathrm{~m}$ (minimum distance between probes to $210 \mathrm{~m}$. 


\section{STUDIES IN PW TUNNEL}

From October 1999 to February 2000 we carried our measurements in the Fermilab PW (Proton West) tunnel.

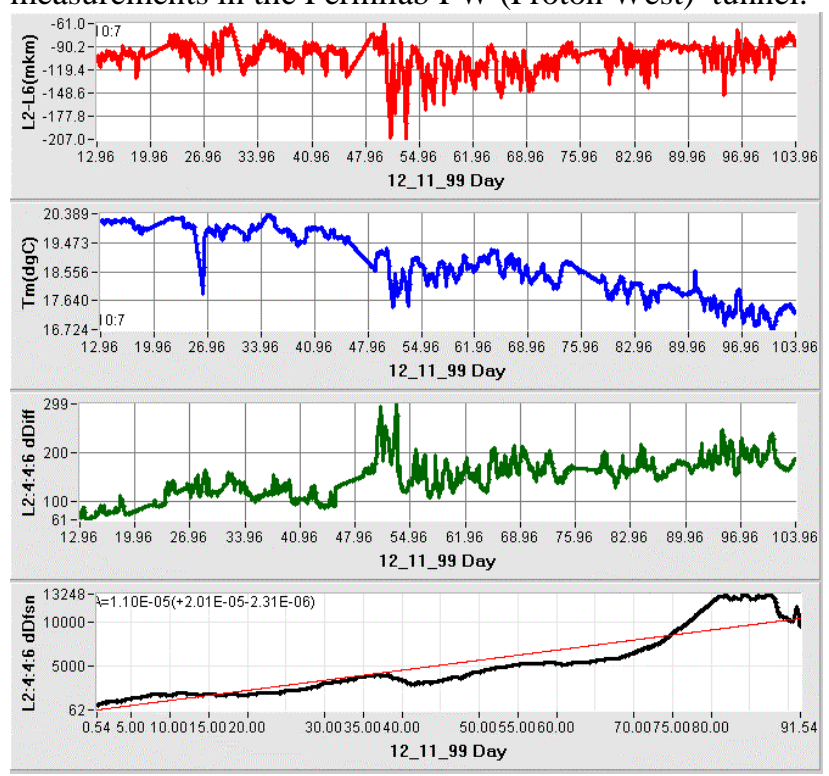

Figure 2: 91 days PW data starting November 12, 1999.

This is an unused beam line for fixed target experiments with a shallow (5 m depth) tunnel built by "cut-andcover" method in 1970's. It has flat concrete floor that made quite easy the installation of 6 HLSs over total length of $180 \mathrm{~m}(30+30+60+30+30$ meter apart). An important drawback of the tunnel was that it was not possible to seal it completely, and there were huge temperature variations from one end to the other. Despite (sometimes, because of) few automatic air conditioning units spread along the tunnel, average temperature also varied a lot - sometimes by few ${ }^{\circ} \mathrm{C}$ a day, and some $4{ }^{\circ} \mathrm{C}$ over $3^{1} \frac{1}{2}$ months (see the second plot from top in Fig.2). Obviously it caused a lot of changes in the level readings, e.g., as big as $150 \mu \mathrm{m}$ are seen in FD26 - sensors $120 \mathrm{~m}$ apart, top plot in Fig. 2 - and about $300 \mu \mathrm{m}$ in SD2446 (the $3^{\text {rd }}$ plot from top). It is not clearly seen in Fig. 2 - and will be demonstrated in the next section - but earth times occur two times a day with some $20 \mu \mathrm{m}$ peak-to-peak amplitude in FD26 but practically absent in SD2446 (and, thus, do not affect accelerators). Power of the latter variation grows approximately linear with time $D \_S D 2446(T) \approx T * 114 \mu \mathrm{m}^{2} /$ day (see dashed line in the bottom plot). There is a handy "ATL law" [4] which say that the variance (mean square) of random ground motion grows linearly with time interval and distance between observation points. For the second difference $Y$ one has:

$$
D \_Y=2 \times A \times T \times L
$$

where $A$ is a coefficient. E.g., for the PW data on $S D 2446$, $L=60 \mathrm{~m}$, and we have $A=1.1 \times 10^{-5} \mu \mathrm{m}^{2} / \mathrm{s} / \mathrm{m}$. Making statistical analysis for all possible combination of probes we get $A=(6.4 \pm 3.6) \times 10^{-6} \mu \mathrm{m}^{2} / \mathrm{s} / \mathrm{m}$. At larger time intervals a systematic ground motion can dominate the random one as we see in the plot of SD2446 which shows some $40 \mu \mathrm{m} /$ month drift up. It was proposed in [5] to separate the random and systematic components as $A_{R}=A_{\mathrm{R}}+A_{\mathrm{S}}=A_{\mathrm{R}}+B T$. To determine natural random contribution, we extracted temperature and systematic linear drifts and got $A_{\mathrm{R}}=(1.2 \pm 0.8) \times 10^{-6} \mu \mathrm{m}^{2} / \mathrm{s} / \mathrm{m}$. From that , we can estimate "transition time" beyond which the linear drifts dominate at $L=30-60 \mathrm{~m}$ as $T=(A$ $\left.A_{\mathrm{R}}\right) / B=24$ days. The lack of data points in spatial intervals does not allow us to confirm or reject the $L$-dependence of the proposed models.

An interesting observation has been made on October 16, 1999 when an earthquake magnitude 7.0 with epicenter at Mojave desert, CA caused some 1200 $\mu \mathrm{m}$ peak-to-peak absolute ground motion at the PW tunnel, and about $F D 06=30 \mu \mathrm{m}$ in the motion of two points $180 \mathrm{~m}$ apart. Simple estimate shows that as much as $30^{2} / 1200=0.75 \mu \mathrm{m}$ of that motion can be transferred into beam-relevant motion of the VLHC quadrupoles.

\section{MEASUREMENTS IN AURORA MINE}

In April 2000, we moved the system of 8 HLSs into the Conco-Western Co. mine (North Aurora, IL) - some 3 miles South-West of Fermilab.

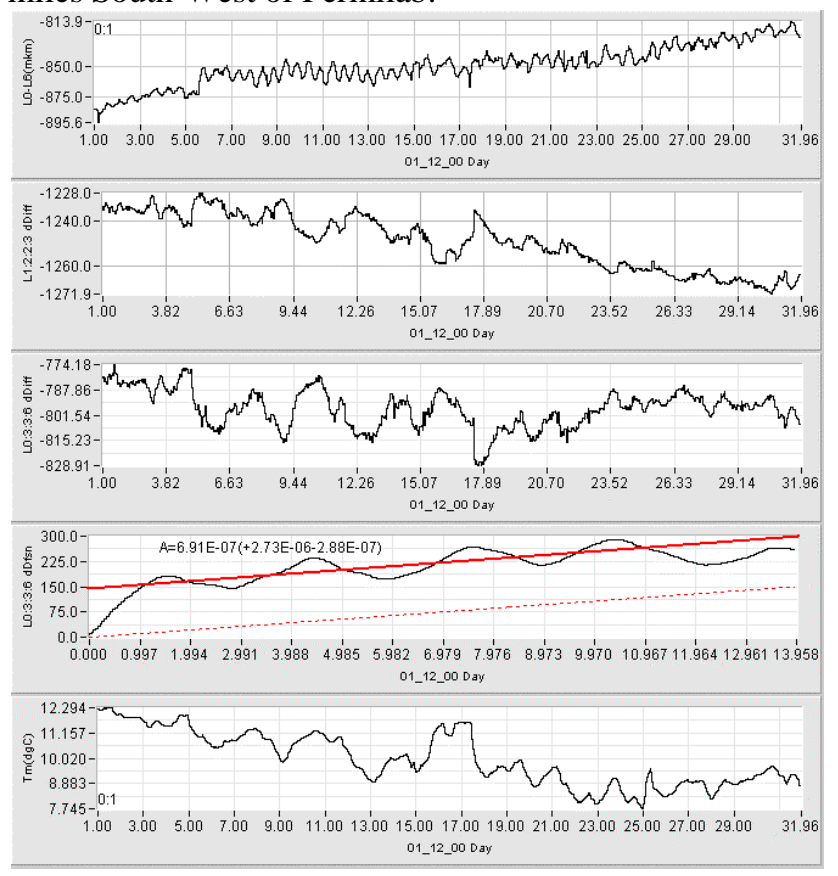

Figure 3: Aurora mine ground motion from December 1 to December 31, 2000.

This is a 200-300 ft deep multi-layer mine in GalenaPlattville dolomite. Our $210 \mathrm{~m}$ long system was set at the depth of $250 \mathrm{ft}$ near the border wall of this $0.8 \mathrm{~km} \times 1.4 \mathrm{~km}$ underground facility. During the studies the mine continued dolomite production and some 3 tons of explosives were detonated each day at around 3 p.m. except weekends in different areas and at different levels of the mine. It is anticipated that the blasting will be moved to the very opposite part of the mine from our system - some $1 \mathrm{~km}$ away - in the fall of 2001. Ventilation system makes the temperature of mine very dependent on the outside temperature. 
The blasts strongly affect our measurements, as they cause jumps in the sensor positions - e.g., see some 20 $\mu \mathrm{m}$ jump up on Dec.6, 2000 and some $15 \mu \mathrm{m}$ jump down and up on Dec.17 in the FD06 record shown in the top plot of Fig. 3 (probes are $180 \mathrm{~m}$ apart). At the same plot one can see $20 \mu \mathrm{m}$ variation two time a day due to tides, and some $80 \mu \mathrm{m}$ drift over 30 days. The second differences SD1223 (probes $30 \mathrm{~m}$ apart) and SD0336 (90 $\mathrm{m}$ apart) presented in the second and the third plots from the top do not contain any visible signs of the tides (amplitude is less than $1 \mu \mathrm{m}$ ) but do show the blasting jumps and the drifts, which often correlate with the average temperature variations (see the bottom plot). Dispersion $D \_S D 0336(T)$ for time intervals up to 14 days is shown in the second plot from the bottom, and can be approximated as $D \_S D 0336(T)\left[\mu^{2}\right]=150+2 A T L$, with $A=6.9 \times 10^{-7} \mu \mathrm{m}^{2} / \mathrm{s} / \mathrm{m}$ (see red line, $L=30 \mathrm{~m}$ ). One can associate excessive dispersion at small time intervals with the blasts. Extraction of temperature correlated signals and linear drifts leads to the average (over all combination of the second differences and over all possible $L=30,60$, $90 \mathrm{~m})$ value of $A_{\mathrm{R}}=(5.8 \pm 2.8) \times 10^{-7} \mu \mathrm{m}^{2} / \mathrm{s} / \mathrm{m}$.

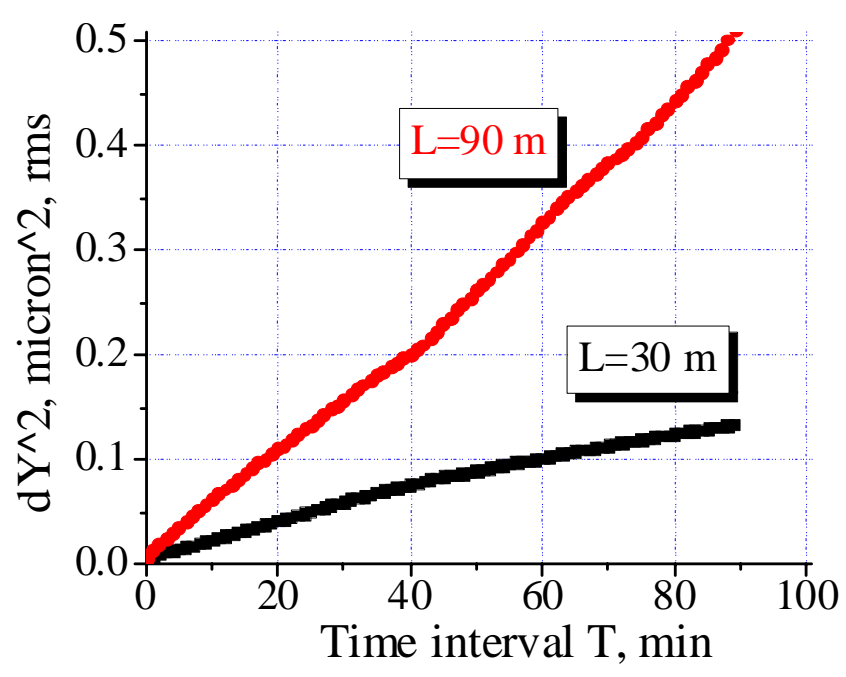

Figure 4: Dispersion of the ground motion for different probe distances, measured on October 13-15, 2000.

There were no blasts over weekends as well as sometimes the temperature does not change much as well, so one can use such records for analyzing "natural" ground diffusion at shorter time scales. For example, on a quiet weekend of Oct. 13-15, 2000, the temperature variation was less than $0.05^{\circ} \mathrm{C}$. The 2 days record analysis is presented in Fig.4 which shows the dispersion of the second differences $D \_S D 1447(T)(L=90 \mathrm{~m}$, red circles $)$ and $D \_S D 1223(T)$ ( $L=30 \mathrm{~m}$, black squares) for up to $T=90$ minutes time intervals. In good accordance with the ATL law, the dispersions grow linearly with the time (coefficients are $A_{\mathrm{R}}=5.3 \times 10^{-7} \mu \mathrm{m}^{2} / \mathrm{s} / \mathrm{m}$. and $A_{\mathrm{R}}=4.2 \times 10^{-7} \mu \mathrm{m}^{2} / \mathrm{s} / \mathrm{m}$, correspondingly), and the dispersion is about 3 times larger for 3 time larger distance.

\section{CONCLUSIONS, DISCUSSION}

We have observed that slow ground motion in shallow PW tunnel is some 10 times more powerful than in $250-\mathrm{ft}$ deep Aurora mine, corresponding ATL-approximation coefficients are $A=(1-10) \times 10^{-6} \mu^{2} / \mathrm{s} / \mathrm{m}$ and $A=(0.2-$ 2) $\times 10^{-6} \mu \mathrm{m}^{2} / \mathrm{s} / \mathrm{m}$. At 1 month time scale temperature related systematic shifts dominate random ground motion. Having thousands data points in time we have statistically proven the ATL-like time dependence of the diffusive ground motion, but 8 HLS probe system can not decisively prove the $L$-dependence. We plan to develop a new system with some 20 new HLSs with order of magnitude smaller noise, and which will employ halffilled water pipes to reduce sensitivity to water temperature variations effects [6].

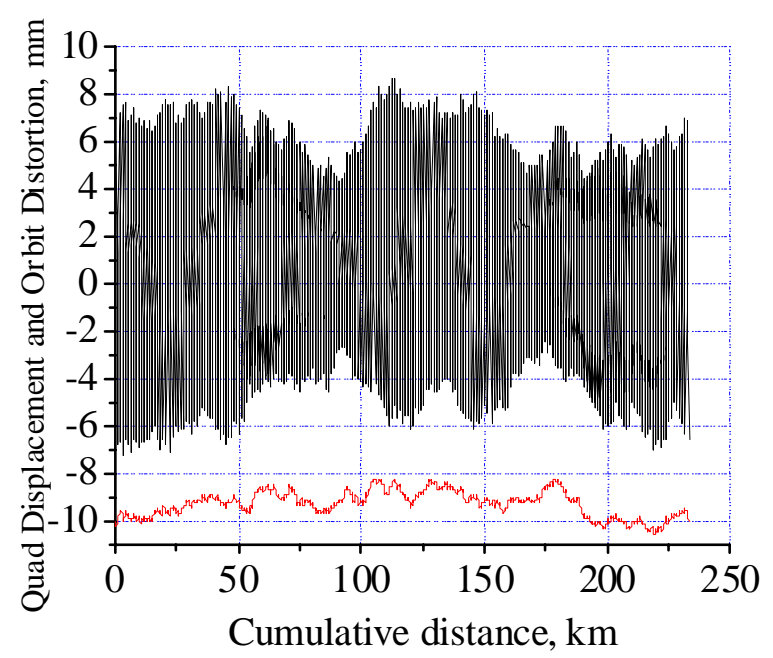

Figure 5: Closed orbit distortion in the VLHC 1 year after initial alignment, $A_{\mathrm{R}}=1 \times 10^{-6} \mu \mathrm{m}^{2} / \mathrm{s} / \mathrm{m}$

Fig.5 shows quadrupole movements in the VLHC over 1 year (bottom curve) and corresponding orbit distortions (some $8 \mathrm{~mm}$ maximum, $1.7 \mathrm{~mm} \mathrm{rms}$ ) calculated under assumption of the ATL law with $A_{\mathrm{R}}=1 \times 10^{-6} \mu \mathrm{m}^{2} / \mathrm{s} / \mathrm{m}$. To keep the orbit in the limits $\pm 9 \mathrm{~mm}$ either regular realignment or $0.2 \mathrm{Tm}$ correctors are needed.

Emittance control in the NLC main linac depends on speed of a beam-based alignment system, and the ground motion observed in the Aurora mine is tolerable for 30min fast system[3], while correction of the PW ground noises would require 10 times faster system.

We are thankful to M.Dunn M.Averett, A.Sery for valuable assistance in our studies.

\section{REFERENCES}

[1] B.Baklakov, et.al, Phys.Rev.ST-AB,1,031001(1998)

[2] VLHC Design Study, FNAL TM-2149 (2001).

[3] NLC ZDR, SLAC Report-474 (1996).

[4] B.Baklakov, et. al, Sov.Tech.Phys, 38 (1998), 894.

[5] A.Sery, T.Raubenheimer, SLAC-Pub-8595 (2000).

[6] A. Chupyra, et.al, these Proceedings. 